\title{
Effect of price elasticity of demand in monopolies with gradient adjustment
}

\author{
Fausto Cavalli ${ }^{1, *}$, Ahmad Naimzada ${ }^{1}$ \\ ${ }^{a}$ Department of Economics, Management and Statistics, University of Milano-Bicocca, U6 \\ Building, Piazza dell'Ateneo Nuovo 1, 20126 Milano, Italy
}

\begin{abstract}
We study a monopolistic market characterized by a constant elasticity demand function, in which the firm technology is described by a linear total cost function. The firm is assumed to be boundedly rational and to follow a gradient rule to adjust the production level in order to optimize its profit. We focus on what happens on varying the price elasticity of demand, studying the effect on the equilibrium stability. We prove that, depending on the relation between the market size and the marginal cost, two different scenarios are possible, in which elasticity has either a stabilizing or a mixed stabilizing/destabilizing effect.
\end{abstract}

Keywords: Boundedly rational monopoly, elasticity, gradient dynamics, stability, bifurcation, complex dynamics

\section{Introduction}

Even if economic theory usually assumes agents to be endowed with perfect rationality, there is a lot of evidence showing that economic agents often have only reduced informational and computational capabilities, in particular when the information set is too broad. In such situations, agents are inclined to use simple decisional heuristics (Kahneman et al. [1]), based on adaptive and learning mechanism in order to approach their optimal choice. To this end, different reduced rationality adjustment mechanisms have been proposed and studied: for a complete dissertation of possible heuristics, we refer to the book of Bischi et al. [2].

Even if a monopolistic market is much more simple than an oligopolistic one, it is likewise realistic to assume "ignorant monopolists", since "ignorance about demand conditions is a ubiquitous feature of market life in the real world" (Clower [3], pp. 716). Both Clower in [3] and later Baumol and Quandt in [4] investigated a monopolistic market in which the firm has reduced rationality,

\footnotetext{
* Corresponding author

Email addresses: fausto.cavalli@unimib.it (Fausto Cavalli), ahmad.naimzada@unimib.it (Ahmad Naimzada)
} 
proposing a rule of thumb mechanism which, under suitable conditions, allows progressively adjusting output levels toward the profit maximizing output. Such mechanisms are based on a gradient rule, namely when the profit variation is positive (resp. negative), the agent tries to adapt his choice increasing (resp. decreasing) it. The adjustment is usually regulated by a multiplicative constant which represents the reaction speed of the economic agent.

Subsequently, Puu in [5] reconsidered the previous framework. Taking into account an economy characterized by a cubic price function with an inflection point, he studied a two dimensional discrete time model in which the monopolist chooses his production level according to a discrete gradient, and he showed the occurrence of complex dynamics and multistability. Subsequently, Naimzada and Ricchiuti in [6] proved that similar complex dynamics are possible considering a simple cubic price function without inflection point, taking into account a continuous gradient rule in a one dimensional time discrete model.

Later researches moved toward the generalization of [5] and [6], considering more and more general demand and cost functions and improving the decisional mechanism, introducing for instance memory and delay, in both continuous and discrete models (Askar in [7], Matsumoto and Szidarowski in [8, 9, 10]). Most of this literature focuses on the effects of the decision-making mechanism on the equilibrium stability, showing that increasing the reaction speed of agents has a destabilizing effect.

About similar models and investigations, we also mention the contributions of Naimzada and Ricchiuti [11], Matsumoto et al. [12, 13], Al-Hdaibat et al. [14].

In our contribution we want to draw attention to the effects of the variation of the demand function on the stability of the steady state and on the convergence toward the profit maximizing output. To this end, we consider an economic setting characterized by a microfunded demand function with constant elasticity $\varepsilon$, and we investigate the effect of the variation of $\varepsilon$ on the steady state, the optimal profit and the local stability of the equilibrium. We assume that the technology of the firm is described by linear total costs. In such scenario, we prove that the steady state is unique and that corresponds to the maximizing profit output.

Our main result consists in proving a dual elasticity behavior. When the marginal cost is small with respect to the market size, we have that increasing the price elasticity of demand has a stabilizing effect on the equilibrium, and there is always a sufficiently large value of elasticity for which the equilibrium is stable and trajectories converge to the maximizing profit output. Conversely, when the marginal cost is large with respect to the market size, we have a mixed scenario. In fact, in this case, equilibrium is unstable for suitably small and large values of elasticity, while it can be, depending on marginal cost, stable in the intermediate interval.

In this work we also investigate the long-term behavior of profits, considering the evolution of cumulative profits, showing that equilibrium profits are larger than profits achieved when equilibrium becomes unstable.

The work is organized as follows: in Section 2 we introduce the model, which 
is then analyzed in Section 3. Section 4 is devoted to simulations. Finally. we propose conclusions and future research aims in Section 5.

\section{Model}

The monopoly model we consider is set in a market characterized by an isoelastic demand function (a microfoundation for a duopoly can be found in the work by Fanti et al. [15])

$$
p(q)=\frac{a}{q^{1 / \varepsilon}}
$$

which, for $\varepsilon>1$ and $a>0$, is strictly decreasing and concave. Moreover, $\varepsilon$ represents the price elasticity of demand. Assuming constant marginal cost $c>0$, the profit function $\pi:(0,+\infty) \rightarrow \mathbb{R}$ is

$$
\pi(q)=q \cdot \frac{a}{q^{1 / \varepsilon}}-c q
$$

and, consequently, the marginal profit function is

$$
\pi^{\prime}(q)=\frac{\varepsilon-1}{\varepsilon} \frac{a}{q^{1 / \varepsilon}}-c .
$$

In what follows, we study the case of $\varepsilon>1$, which allows the profit function (2) to have a unimodal shape. The remaining case of inelastic or unitary elastic demands $(\varepsilon \leq 1)$ can not be handled with the approach we consider, as in such case the profit function would be decreasing, leading to $q \rightarrow 0$ optimal choice. This is an economically uninteresting situation in which, moreover, $\pi \rightarrow+\infty$.

As in [6], we suppose that the monopolist, who has a reduced degree of rationality, in order to maximize his profit, tries to adapt his production level following a "rule of thumb" mechanism, increasing (resp. decreasing) the produced quantity when profit variations are positive (resp. negative). This can be modeled through a gradient mechanism, in which the variation of $q$ from time $t$ to $t+1$ is proportional to the variation of profits induced by the production level at time $t$, namely

$$
q_{t+1}-q_{t}=\alpha \pi^{\prime}\left(q_{t}\right),
$$

in which $\alpha>0$ represents the reactivity (or adjustment speed) of the agent.

The resulting adjustment mechanism is then described by the discrete dynamical system

$$
q_{t+1}=q_{t}+\alpha\left(\frac{\varepsilon-1}{\varepsilon} \frac{a}{q^{1 / \varepsilon}}-c\right)
$$

which, after having introduced parameters $k=\alpha a$ and $d=c / a$, can be rewritten as

$$
q_{t+1}=f\left(q_{t}\right)=q_{t}+k\left(\frac{\varepsilon-1}{\varepsilon} \frac{1}{q^{1 / \varepsilon}}-d\right) .
$$


We remark that starting from an initial production level $q_{0}>0$, trajectories $q_{t}$ may cross unfeasible production levels regions $q_{t} \leq 0$. In this work, we will only focus on such initial data and parameters for which the output level stay positive for all $t$. Conversely, when $f\left(q_{t}\right) \leq 0$, the next time production level should be set to zero.

In the next proposition, we study the behavior of function $f$.

Proposition 1. Function $f(q):(0,+\infty) \rightarrow \mathbb{R}$, defined by (5), is decreasing for $q<q_{m}$ and increasing for $q>q_{m}$, where

$$
q_{m}=\left(k \frac{(\varepsilon-1)}{\varepsilon^{2}}\right)^{\frac{\varepsilon}{\varepsilon+1}}
$$

is its (global) minimum point, for which we have

$$
f\left(q_{m}\right)=\left(k \frac{\varepsilon-1}{\varepsilon^{2}}\right)^{\frac{\varepsilon}{\varepsilon+1}}+k\left(\frac{\varepsilon-1}{\varepsilon}\left(\frac{\varepsilon^{2}}{k(\varepsilon-1)}\right)^{\frac{1}{\varepsilon+1}}-d\right) .
$$

Proof. Thesis easily follows from the derivative of $f$

$$
f^{\prime}(q)=1-\frac{k(\varepsilon-1)}{\varepsilon^{2} q^{(\varepsilon+1) / \varepsilon}}
$$

by imposing $f^{\prime}(q)>0$.

The previous proposition assures that $f$ is non negative provided that $f\left(q_{m}\right) \geq$ 0 . In particular, we remark that since the only negative term in $f\left(q_{m}\right)$ is $-d$, positivity is guaranteed provided that the marginal cost $c$ is sufficiently small with respect to the market size $a$.

In addition to the positivity of the output, we also need to check the positivity of profit $\pi\left(q_{t}\right)$, since, from (2), profit is positive provided that $q \geq d^{-\varepsilon}$. Although it is acceptable that profit becomes negative for short times, cumulative profits, namely the sum of profits over times, have to stay positive. Supposing that the monopolist have an initial endowment $\Pi_{0}$, we can recursively define cumulative profits $\Pi_{t}$ through

$$
\Pi_{t+1}=\Pi_{t}+\pi\left(q_{t+1}\right), \quad t>0 .
$$

In general, it is not possible to compute analytically $\Pi_{t}$. In Section 4 , cumulative profits are computed numerically and their behavior is studied through simulations. Similar analysis is performed by Matsumoto in $[16,17,18]$.

\section{Analysis}

Before studying analytically the possible steady states of (5) and their local stability, we want to make some remarks. The steady state clearly requires that the marginal profit vanishes, which means that the marginal revenue

$$
R_{a, \varepsilon}(q)=\frac{\varepsilon-1}{\varepsilon} \frac{a}{q^{1 / \varepsilon}}
$$


must equal the marginal cost $c$. Function $R_{a, \varepsilon}(q)$ has a hyperbolic shape, since

$$
\lim _{q \rightarrow 0^{+}} R_{a, \varepsilon}(q)=+\infty, \lim _{q \rightarrow+\infty} R_{a, \varepsilon}(q)=0
$$

and $R_{a, \varepsilon}^{\prime}(q)<0, R_{a, \varepsilon}^{\prime \prime}(q)>0$, and it can be very steep for $q<\tilde{q}$ and very flat $q>\tilde{q}$, where $\tilde{q}$ is such that $\left|R_{a, \varepsilon}^{\prime}(\tilde{q})\right|=1$. Moreover, as elasticity $\varepsilon$ increases, such behavior becomes more and more pronounced. It is easy to see that

$$
\tilde{q}=\left(a \frac{\varepsilon-1}{\varepsilon^{2}}\right)^{\varepsilon /(\varepsilon+1)},
$$

to which corresponds

$$
R_{a, \varepsilon}(\tilde{q})=\frac{\varepsilon-1}{\varepsilon}\left(\frac{\varepsilon^{2}}{\varepsilon-1}\right)^{1 /(\varepsilon-1)} a^{\varepsilon /(\varepsilon+1)} .
$$

Since

$$
\lim _{\varepsilon \rightarrow+\infty} \frac{\varepsilon-1}{\varepsilon}\left(\frac{\varepsilon^{2}}{\varepsilon-1}\right)^{1 /(\varepsilon-1)}=1,
$$

we have that

$$
\lim _{\varepsilon \rightarrow+\infty} R(\tilde{q})=a .
$$

This means that, with respect to the price elasticity of demand, we can expect two different asymptotic scenarios. When marginal cost $c$ is larger than $a$, for sufficiently large $\varepsilon$ the equilibrium will fall in the region in which the marginal revenue function is very steep. Since the local stability of the equilibrium depends on the marginal revenue variation, we can expect that when $c>a$ elasticity has a destabilizing effect. Conversely, if marginal cost $c$ is smaller than $a$, for sufficiently large $\varepsilon$ the equilibrium will fall in the region in which the marginal revenue function is very flat, and in this case we can expect that elasticity has a stabilizing effect.

To check the previous conjecture, we start studying the possible steady states of (5).

Proposition 2. When $\varepsilon>1$, dynamical system (5) has a unique steady state

$$
q^{*}=\left(\frac{\varepsilon-1}{\varepsilon d}\right)^{\varepsilon},
$$

in which profit function (2) attains its maximum. The corresponding equilibrium values for price and profit are

$$
p^{*}=c \frac{\varepsilon}{\varepsilon-1}
$$

and

$$
\pi^{*}=\left(\frac{a}{\varepsilon}\right)^{\varepsilon}\left(\frac{\varepsilon-1}{c}\right)^{\varepsilon-1} .
$$


Proof. Value $q^{*}$ is found by imposing $f(q)=q$, which reduces to the first order condition for profits $\pi^{\prime}(q)=0$, which is solved by (10). Since

$$
\pi^{\prime \prime}(q)=-\frac{k(\varepsilon-1)}{\varepsilon^{2} q^{1 / \varepsilon+1}}<0
$$

second order condition is satisfied. Equilibrium values (11) and (12) can be easily computed.

The previous proposition states that the steady state of system (5) is unique. Now, we analyze how equilibrium values change on varying elasticity $\varepsilon$.

Looking at the behavior of the equilibrium production level, if we compute $\partial_{\varepsilon} q^{*}$

$$
\partial_{\varepsilon} q^{*}=\left(\frac{\varepsilon-1}{d \varepsilon}\right)^{\varepsilon}\left(\frac{1}{\varepsilon-1}-\log \frac{\varepsilon}{\varepsilon-1}-\log (d)\right),
$$

we have that $(1 /(\varepsilon-1)-\log (\varepsilon) /(\varepsilon-1))$ is a strictly decreasing positive function vanishing for $\varepsilon \rightarrow+\infty$. This means that, when $d \leq 1$, we have $\partial_{\varepsilon} q^{*}>0$ and $q^{*}$ increases with the elasticity. Conversely, for each $d>1$, there exists a $\varepsilon_{d}$ such that $\partial_{\varepsilon} q^{*}\left(\varepsilon_{d}, d\right)=0$, so $q^{*}$ increases when $\varepsilon$ is increased from 1 to $\varepsilon_{d}$, while it decreases when $\varepsilon$ is increased above $\varepsilon_{d}$, hence attaining its maximum when elasticity is $\varepsilon_{d}$. This behavior is reported in Figure 1 .
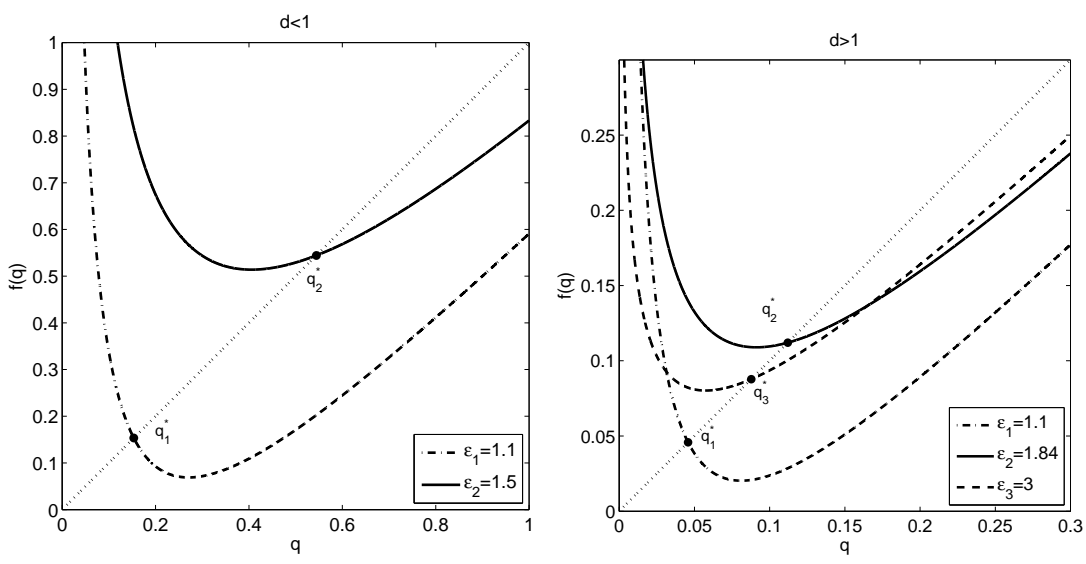

Figure 1: Plot of $f$ for different values of parameters. Left plot: we set $d=0.5$ and $k=1$ and we considered two different elasticity values $\varepsilon_{1}=1.1$ and $\varepsilon_{2}=1.5$. The equilibrium output level increases with $\varepsilon$. Right plot: we set $d=1.5$ and $k=0.1$ and we considered three different elasticity values $\varepsilon_{1}=1.1, \varepsilon_{2}=\varepsilon_{d} \approx 1.84$ and $\varepsilon_{3}=3$, where $\varepsilon_{d}$ is the solution of $\partial_{\varepsilon} q^{*}=0$. The equilibrium production level increases with $\varepsilon$ for $\varepsilon<\varepsilon_{d}$, then it decreases.

To investigate the variation of $\pi^{*}$ with respect to $\varepsilon$, we compute $\partial_{\varepsilon} \pi^{*}$

$$
\partial_{\varepsilon} \pi^{*}=-\frac{c(\varepsilon-1)^{\varepsilon-1}(\log (\varepsilon)-\log (\varepsilon-1)+\log (d))}{\varepsilon^{\varepsilon} d^{\varepsilon}} .
$$


We have that $\partial_{\varepsilon} \pi^{*}<0$ for any $\varepsilon>1$ when $d \geq 1$, while, for $d<1$, we have $\partial_{\varepsilon} \pi^{*}<0$ when $\varepsilon<d /(d+1)$ and $\partial_{\varepsilon} \pi^{*}>0$ when $\varepsilon>d /(d+1)$. In the former case, we have that when elasticity increases, equilibrium profit decreases, while in the latter case, we have that equilibrium profit attains its minimum when $\varepsilon=d /(d+1)$.

Finally, equilibrium price $p^{*}$ is strictly decreasing with respect to $\varepsilon$.

The previous results confirm the possibly ambiguous behavior of (5) with respect to the price elasticity of demand. As conjectured, the model differently acts depending on the ratio between the marginal cost and the market size. In particular, we have two opposite behaviors when elasticity is sufficiently large, as if $\varepsilon$ is sufficiently small a positive variation of elasticity always leads to larger equilibrium production levels and smaller equilibrium profits for both $d \leq 1$ and $d>1$. Conversely, if $\varepsilon$ is sufficiently large, when $d<1$ an increase in the elasticity again corresponds to an increase in the equilibrium production level, but, at the same time, it results in increasingly large profits for $\varepsilon>d /(d+1)$, while when $d>1$ both $q^{*}$ and $\pi^{*}$ reduce. This means that if marginal cost is relatively small, since $\pi^{*} \rightarrow+\infty$ for $\varepsilon \rightarrow+\infty$, the equilibrium profits are large in those markets characterized by large elasticity values. Conversely, if $d>1$, large profits are achieved only for $\varepsilon \rightarrow 1$.

As we said, in Section 4, we investigate through simulations the behavior of cumulative profits. As a basis for comparison, we can consider cumulative profits that the monopolist would achieve if he always chose equilibrium $q^{*}$, i.e. cumulative equilibrium profits

$$
\Pi_{t+1}^{*}=\Pi_{t}^{*}+q^{*} p^{*}-c q^{*}=\Pi_{0}+(t+1) \pi^{*}=\Pi_{0}+(t+1)\left(\frac{a}{\varepsilon}\right)^{\varepsilon}\left(\frac{\varepsilon-1}{c}\right)^{\varepsilon-1} .
$$

In the next propositions we study the local stability of the steady state with respect to elasticity $\varepsilon$. Since different scenarios arise, we split the analysis into two parts, considering separately $d \leq 1$ and $d>1$.

Proposition 3. For each $0<d \leq 1$ and $k>0$, there exists $\tilde{\varepsilon}(d, k)$ such that $q^{*}$ is locally asymptotically stable for $\varepsilon>\tilde{\varepsilon}(d, k)$ and unstable for $1<\varepsilon<$ $\tilde{\varepsilon}(d, k)$. Moreover, stability threshold $\tilde{\varepsilon}(d, k)$ increases with both $k$ and $d$. A flip bifurcation occurs for $\varepsilon=\tilde{\varepsilon}(d, k)$.

Proof. Equilibrium $q^{*}$ is stable provided that

$$
-1<f^{\prime}\left(q^{*}\right)<1
$$

where

$$
f^{\prime}\left(q^{*}\right)=1-k \frac{\varepsilon-1}{\varepsilon^{2}}\left(\frac{d \varepsilon}{\varepsilon-1}\right)^{\varepsilon+1}
$$

Inequality $f^{\prime}\left(q^{*}\right)<1$ is indeed true, to study $f^{\prime}\left(q^{*}\right)>-1$ we set $\varepsilon=1 / \gamma$, and we have to solve

$$
k \gamma(1-\gamma)\left(\frac{d}{1-\gamma}\right)^{1 / \gamma+1}<2
$$


for $\gamma \in(0,1)$. Computing the logarithm of both sides, we can rewrite the last inequality as

$$
z_{k, d}(\gamma)=\log (k)+\log (\gamma)+\left(1+\frac{1}{\gamma}\right) \log (d)-\frac{1}{\gamma} \log (1-\gamma)<\log (2)
$$

where

$$
\lim _{\gamma \rightarrow 0^{+}} z_{k, d}(\gamma)=-\infty, \quad \lim _{\gamma \rightarrow 1^{-}} z_{k, d}(\gamma)=+\infty
$$

The two previous limits assure the existence of at least a solution $\tilde{\gamma}$ of $z_{k, d}(\gamma)=$ $\log 2$, while uniqueness is guaranteed by the strict monotonicity of $z(\gamma)$, namely by

$$
z_{k, d}(\gamma)^{\prime}=\frac{2}{\gamma}+\frac{1}{1-\gamma}-\frac{1}{\gamma^{2}} \log (d)+\frac{1}{\gamma^{2}} \log (1-\gamma)>0
$$

To prove (15), we notice that the first three terms are positive, while the last one is negative. We will deal with two cases separately, $\gamma \in\left(0,1-e^{-1}\right]$ and $\gamma \in\left(1-e^{-1}, 1\right)$. In the first interval, since $2 \gamma>-\log (1-\gamma)$, we have

$$
\frac{2}{\gamma}>-\frac{1}{\gamma^{2}} \log (1-\gamma),
$$

and then $z_{k, d}^{\prime}(\gamma)>0$ for $\gamma \in\left(0,1-e^{-1}\right]$. Furthermore, for $\gamma \in\left(1-e^{-1}, 1\right)$, we can prove that $\gamma^{-2} \log (1-\gamma)$ is dominated by $1 /(1-\gamma)$, or, equivalently,

$$
\zeta(\gamma)=\gamma^{2}+(1-\gamma) \log (1-\gamma)>0
$$

Since $\zeta\left(1-e^{-1}\right)=1+e^{-2}-3 e^{-1} \approx 0.0317>0$ and, for $\gamma \in(1 / 2,1)$,

$$
\zeta^{\prime}(\gamma)=2 \gamma-1-\log (1-\gamma)>0,
$$

we can conclude that $\zeta(\gamma)>0$ for all $\gamma \in\left[1-e^{-1}, 1\right)$ and so (16) is true.

This proves that $f^{\prime}\left(q^{*}\right)>-1$ for $\gamma \in(0, \tilde{\gamma})$. Setting $\tilde{\varepsilon}=1 / \tilde{\gamma}$ allows concluding that the equilibrium is stable for $\varepsilon>\tilde{\varepsilon}$ and, since $f(\tilde{\varepsilon})=-1$, that the destabilization occurs through a flip bifurcation.

Finally, considering $d_{1} \leq d_{2}<1$ and $k_{1} \leq k_{2}$, from (14), we have that $z_{d_{1}, k_{1}}(\gamma) \leq z_{d_{2}, k_{2}}(\gamma)$ for every $\gamma \in(0,1)$ and, consequently, $z_{k_{i}, d_{i}}(\gamma)=\log 2$ for $i=1,2$ are respectively solved by $\tilde{\gamma}_{2} \leq \tilde{\gamma}_{1}$. Setting $\tilde{\varepsilon}_{i}=1 / \tilde{\gamma}_{i}$, we can conclude.

Previous proposition asserts that, if marginal cost $c$ is sufficiently small with respect to market size $a(c \leq a)$, the price elasticity of demand has a stabilizing effect. Moreover, if marginal cost $c$ or reaction speed $k$ are increased, as well as if market size $a$ is decreased, the stability interval becomes smaller.

Now we focus on the remaining case $d>1$.

Proposition 4. Let be $d>1$ and $k>0$, then two situations are possible:

- there exist $\tilde{\varepsilon}_{A}(d, k)<\tilde{\varepsilon}_{B}(d, k)$ such that $q^{*}$ is locally asymptotically stable for $\varepsilon \in\left(\tilde{\varepsilon}_{A}(d, k), \tilde{\varepsilon}_{B}(d, k)\right)$ and unstable otherwise; 
- the equilibrium is unstable for any $\varepsilon>1$.

In the former case, for both $\varepsilon=\tilde{\varepsilon}_{A}(d, k)$ and $\varepsilon=\tilde{\varepsilon}_{B}(d, k)$, a flip bifurcation occurs. Moreover, if $1<d_{1} \leq d_{2}$ and $k_{1} \leq k_{2}$, the stability region given by $\left(d_{2}, k_{2}\right)$ is a subset of that given by $\left(d_{1}, k_{1}\right)$.

Proof. Imposing stability condition $-1<f^{\prime}\left(q^{*}\right)<1$ and acting as in the proof of Proposition 3, we consider $z_{k, d}(\gamma)$ defined by (14). If $d>1$, we have that

$$
\lim _{\gamma \rightarrow 0^{+}} z_{k, d}(\gamma)=+\infty, \quad \lim _{\gamma \rightarrow 1^{-}} z_{k, d}(\gamma)=+\infty,
$$

so, in this case, the set of values $\gamma$ that satisfy $z_{k, d}>\log (2)$ could be empty. To this end, we study the monotonicity of $z_{k, d}(\gamma)$ by imposing that $z_{k, d}^{\prime}(\gamma)>0$, or, equivalently,

$$
\varphi(\gamma)=2 \gamma+\frac{\gamma^{2}}{1-\gamma}-\log (d)+\log (1-\gamma)>0 .
$$

Since

$$
\lim _{\gamma \rightarrow 0^{+}} 2 \gamma+\frac{\gamma^{2}}{1-\gamma}-\log (d)+\log (1-\gamma)=-\log (d)<0
$$

and

$$
\varphi^{\prime}(\gamma)=\frac{\gamma^{2}-\gamma+1}{(1-\gamma)^{2}}>0
$$

we have that $z^{\prime}(\gamma)$ is strictly increasing, negative for $\gamma<\gamma_{m}$ and positive for $\gamma>\gamma_{m}$, and hence $z$ attains its unique minimum for $\gamma=\gamma_{m}$. Then, if $z\left(\gamma_{m}\right)<\log (2)$, there exist $\tilde{\gamma}_{B}<\tilde{\gamma}_{A}$ so that $z_{k, d}(\gamma)>\log (2)$ if and only if $\gamma \in\left(\tilde{\gamma}_{B}, \tilde{\gamma}_{A}\right)$. Setting $\tilde{\varepsilon}_{A}=1 / \tilde{\gamma}_{B}$ and $\tilde{\varepsilon}_{B}=1 / \tilde{\gamma}_{A}$ we obtain the first stability scenario for $d \geq 1$.

Conversely, if $z_{d, k}\left(\gamma_{m}\right) \geq \log (2)$, we have that (14) is never satisfied and, consequently, $f^{\prime}\left(q^{*}\right) \leq-1$ for all $\varepsilon>1$.

Considering $1<\bar{d}_{1} \leq d_{2}$ and $k_{1} \leq k_{2}$, from (14), we have that the set of $\gamma$ values that solve $z_{d_{2}, k_{2}}(\gamma) \leq \log (2)$ solve $z_{d_{1}, k_{1}}(\gamma) \leq \log (2)$ too, and this allows concluding.

This second scenario is quite different from that depicted in Proposition 3. In fact, for $c>a$, elasticity can have a mixed behavior. If $k, d$ are suitably small, so that the stability region is not an empty set, increasing elasticity $\varepsilon$ has an initial stabilizing effect. However, if $\varepsilon$ is further increased, the equilibrium becomes unstable.

Propositions 3 and 4 prove the different effect on the local stability, depending on $d$, of elasticity variations. Since for both $d \leq 1$ and $d>1$, when $\varepsilon$ approaches 1 the dynamics are inclined to become unstable, cautious adjustment of production levels should be adopted, as decreasing reaction speed $\alpha$ extends the stability region, leading to convergent trajectories for smaller elasticity values too. We notice that if the dynamic does not converge to the equilibrium production level, smaller profits are achieved, as we will show in next section 
simulations, so, in order to achieve the largest possible profits, output levels should be adjusted toward $q^{*}$ as fast as possible. Conversely, if the demand is sufficiently elastic and the marginal cost relatively small, a suitably reactive adjustment mechanism could be more beneficial. In fact, provided that $\alpha$ be suitable to guarantee stable dynamics, the production levels would more quickly approach the profit maximizing output $q^{*}$, allowing for larger cumulative profits. On the contrary, such less cautious behavior is not recommended in the case of relatively large marginal costs, as if demand is very elastic, unstable dynamics may arise one more time. When $d>1$, the behavior should be more and more cautious as $\varepsilon$ increases.

\section{Simulations}

In this section we present some simulations to confirm the theoretical analysis of the previous section and to investigate the scenarios arising when stability conditions are violated. In all the following simulations we set $q_{0}=0.1$ and $\alpha=1$.

Setting $a=1, c=0.9$, we investigate the case of $d<1$, in which, according to Proposition 3, elasticity has a stabilizing role. For such market size and marginal cost, $f_{\varepsilon}^{\prime}\left(q^{*}\right)$ defined in (13), is strictly increasing with respect to $\varepsilon$, as shown in Figure 2. Computing numerically the solution of $f_{\varepsilon}^{\prime}\left(q^{*}\right)=-1$, we

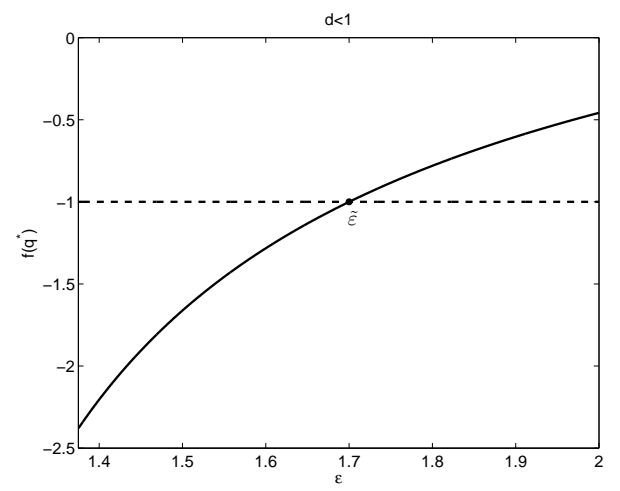

Figure 2: Plot of $f_{\varepsilon}^{\prime}\left(q^{*}\right)$ on varying $\varepsilon$ for $a=1, c=0.9$. Equilibrium $q^{*}$ is stable for $\varepsilon>\tilde{\varepsilon} \approx$ 1.700

obtain the instability threshold $\tilde{\varepsilon} \approx 1.700$, below which equilibrium loses stability through a flip bifurcation. In Figure 3 we report the bifurcation diagram of (5) with respect to $\varepsilon$, which confirms that, starting from a chaotic attractor $(\varepsilon=1.375)$, a sequence of period halving leads to stability when $\varepsilon \approx 1.7$. In Figure 4 we investigate cumulative profits. In the first plot, we show cumulative profits $\Pi_{t}$ for $t \in(8000,10000)$, having set $\varepsilon=2$. Cumulative profits are positive and qualitatively increasing. In the second plot, we report cumulative profits for $T=10000$, for $\varepsilon$ which varies in $(1.375,2)$, together with cumulative 


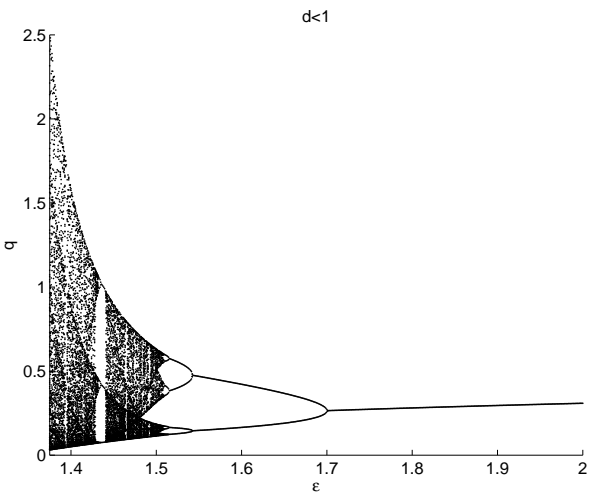

Figure 3: Bifurcation diagram for $d<1$, for $\varepsilon \in(1.375,2)$. The equilibrium is stable for $\varepsilon>1.700$, below which a flip bifurcation occurs.

equilibrium profits. Cumulative profits are positive and coincide with equilibrium cumulative profits for $\varepsilon>\tilde{\varepsilon}$. When the equilibrium becomes unstable, cumulative profits are smaller than those achieved at the equilibrium.
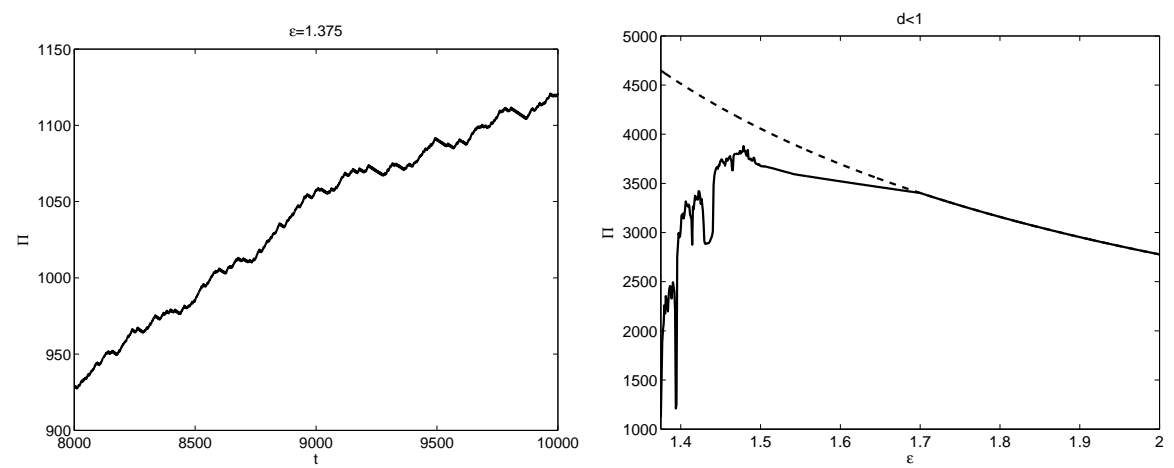

Figure 4: Cumulative profits plots for $d<1$. (Left plot) Cumulative profits for $\varepsilon=0.2$. (Right plot) Solid line: cumulative profits $\Pi_{10000}$ on varying $\varepsilon \in(1.375,2)$. Dashed line: corresponding cumulative equilibrium profits $\Pi_{10000}^{*}$. When equilibrium is stable, $\Pi_{10000}=$ $\Pi_{10000}^{*}$, while below stability threshold we have $\Pi_{10000}<\Pi_{10000}^{*}$.

If we consider $a=0.1, c=0.2$, we are in the scenario studied in Proposition 4 , in which the elasticity has a mixed role. In this case, $f_{\varepsilon}^{\prime}\left(q^{*}\right)$ defined in (13), is unimodal and intersects stability threshold -1 in two distinct points $\tilde{\varepsilon}_{A}$ and $\tilde{\varepsilon}_{B}$, as we can see in Figure 5. Computing numerically such values, we obtain $\tilde{\varepsilon}_{A} \approx 1.478$ and $\tilde{\varepsilon}_{B} \approx 3.356$. Outside interval $(1.478,3.356)$, the equilibrium is unstable, and we have two flip bifurcations, as shown in Figure 6. Starting from $\varepsilon=1.2$, we initially have a period halving that leads to stability when 


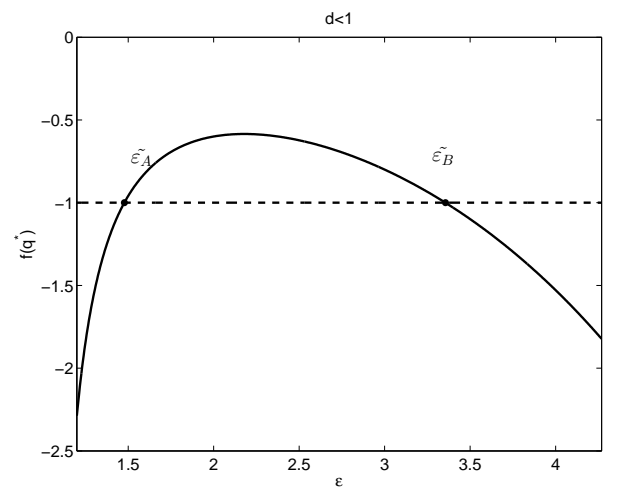

Figure 5: Plot of $f_{\varepsilon}^{\prime}\left(q^{*}\right)$ on varying $\varepsilon$ for $a=0.1, c=0.2$. Equilibrium $q^{*}$ is stable for $\varepsilon \in(1.478,3.356)$

$\varepsilon=\tilde{\varepsilon}_{A}$; then, a period doubling occurs for $\varepsilon=\tilde{\varepsilon}_{B}$, leading again to chaos. In Figure 7 , for $t \in(8000,10000)$ we report cumulative profits $\Pi_{t}$ corresponding to $\varepsilon=4.269$, which stay positive. In the second plot, we report cumulative profits for $\varepsilon \in(1.2,4.269)$ for $T=10000$, together with cumulative equilibrium profits. We have that, outside the stability interval, cumulative profits are smaller than equilibrium cumulative profits again. We remark that cumulative profits behavior we showed is quite predictable in the present case, as, being the profit function unimodal, we have a unique internal equilibrium which provides a global maximum profit. When equilibrium loses its stability, $q_{t}$ converges to attractors which consist of a set of output levels for which profits are always smaller than those achieved for $q=q^{*}$, as shown in Figure 8 .

As noticed in Proposition 4, the stability interval can be empty. For example, slightly modifying the previous parameters and setting $a=0.1, c=0.23$, we can see from Figure 9 that function $f^{\prime}\left(q^{*}\right)<-1$, so equilibrium is unstable for all $\varepsilon>1$. In such configuration, we can again say that elasticity has initially a "qualitatively" stabilizing effect, since, increasing $\varepsilon$ allows for more regular dynamics, as the initial chaotic behavior (5) evolves through a period halving toward a period 2-cycle $(\varepsilon \approx 1.77)$. However, trajectories of system $(5)$ never converge to equilibrium and increasing further elasticity above $\varepsilon \approx 2.176 \mathrm{a}$ cascade of period doubling leads to chaos again. Moreover, increasing further the marginal cost, the intermediate periodic attractors disappear and we just have a chaotic behavior with possible windows of periodicity, as, for example, considering $c=0.24$. The corresponding bifurcation diagrams are reported in Figure 10.

\section{Conclusions}

In our contribution, we investigated the effect of elasticity variation on the local stability of the equilibrium. The main result concerns the existence of two 

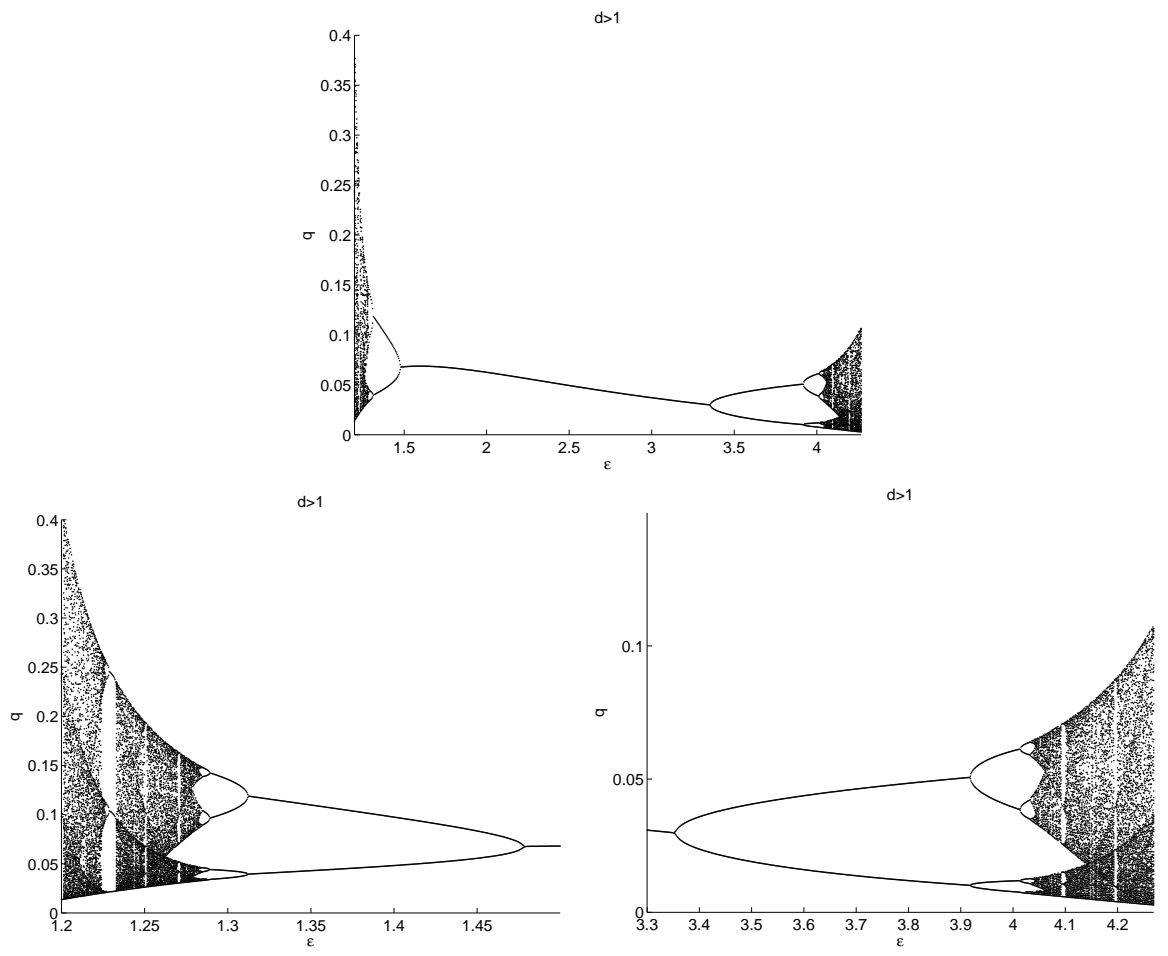

Figure 6: (Top) Bifurcation diagram for $a=0.1, c=0.2$, on varying $\varepsilon \in(1.2,4.269)$. The equilibrium is stable for $\varepsilon \in(1.478,3.356)$. (Bottom, left plot) Blow up of the period halving bifurcation. (Bottom, right plot) Blow up of the period doubling bifurcation.

different behaviors, depending on the relation between the market size and the marginal cost of the firm. When the market size is sufficiently large with respect to the marginal cost, increasing the price elasticity of demand stabilizes the equilibrium, while in the opposite case we have that, even if an initial increase of the elasticity can introduce stability, if the price function becomes too elastic, equilibrium again loses its stability. In particular, in both cases, we never have a scenario of unconditional stability with respect to elasticity. We showed this, by considering a discrete adjustment process, in which the boundedly rational firm adapts its production level following a gradient rule. We aim to investigate the robustness of such result with respect to the decisional mechanism and to the control variable, considering for example an adjustment mechanism for prices.

\section{References}

[1] Kahneman D, Slovic A, Tversky A. Judgment under Uncertainty. Heuristics and Biases. Cambridge University Press; 1982. 

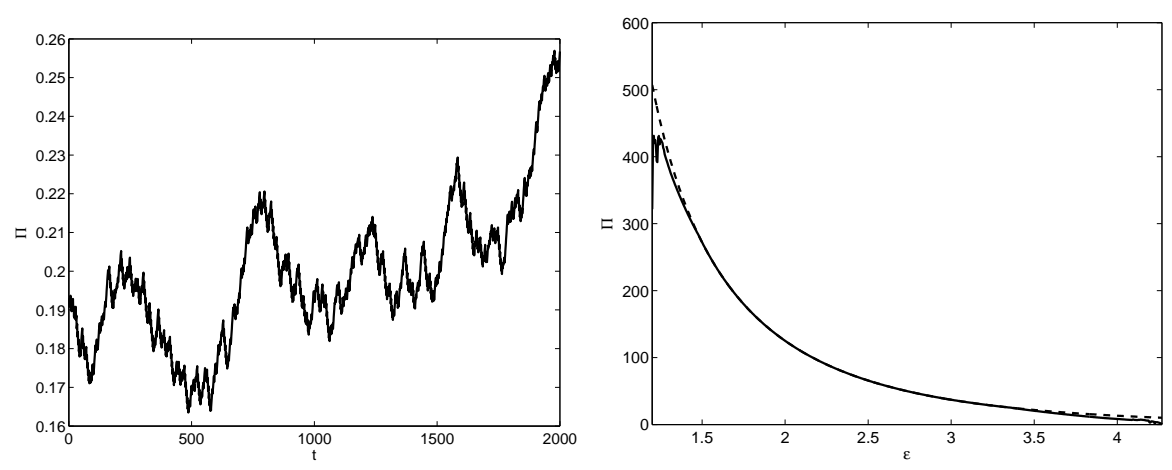

Figure 7: Cumulative profits plots for $a<0.1, c=0.2$. (Left plot) Cumulative profits for $\varepsilon=4.269$, which stay positive. (Right plot) Solid line: cumulative profits $\Pi_{10000}$ on varying $\varepsilon \in(1.2,4.269)$. Dashed line: corresponding cumulative equilibrium profits $\Pi_{10000}^{*}$. When equilibrium is stable, we have $\Pi_{10000}=\Pi_{10000}^{*}$, while below stability threshold we have $\Pi_{10000}<\Pi_{10000}^{*}$.

[2] Bischi GI, Chiarella C, Kopel M, Szidarovsky F. Nonlinear oligopolies: stability and bifurcations. Springer; 2009.

[3] Clower RW. Some theory of an ignorant monopolist. Econ J 1959;69(276):pp. 705-716.

[4] Baumol WJ, Quandt RE. Rules of thumb and optimally imperfect decisions. Am Econ Rev 1964;54(2):23-46.

[5] Puu T. The chaotic monopolist. Chaos, Solitons Fractals 1995;5(1):35-44.

[6] Naimzada A, Ricchiuti G. Complex dynamics in a monopoly with a rule of thumb. Appl Math Comput 2008;203(2):921-5.

[7] Askar SS. On complex dynamics of monopoly market. Econ Model 2013;31(1):586-9.

[8] Matsumoto A, Szidarovszky F. Discrete and continuous dynamics in nonlinear monopolies. Appl Math Comput 2014;232:632-42.

[9] Matsumoto A, Szidarovszky F. Discrete-time delay dynamics of boundedly rational monopoly. Decis Econ Financ 2014;37(1):53-79.

[10] Matsumoto A, Szidarovszky F. Complex dynamics of monopolies with gradient adjustment. Econ Model 2014;42:220-9.

[11] Naimzada A, Ricchiuti G. Monopoly with local knowledge of demand function. Econ Model 2011;28(1-2):299-307.

[12] Matsumoto A, Szidarovszky F. Nonlinear delay monopoly with bounded rationality. Chaos, Solitons Fractals 2012;45(4):507 -19. 


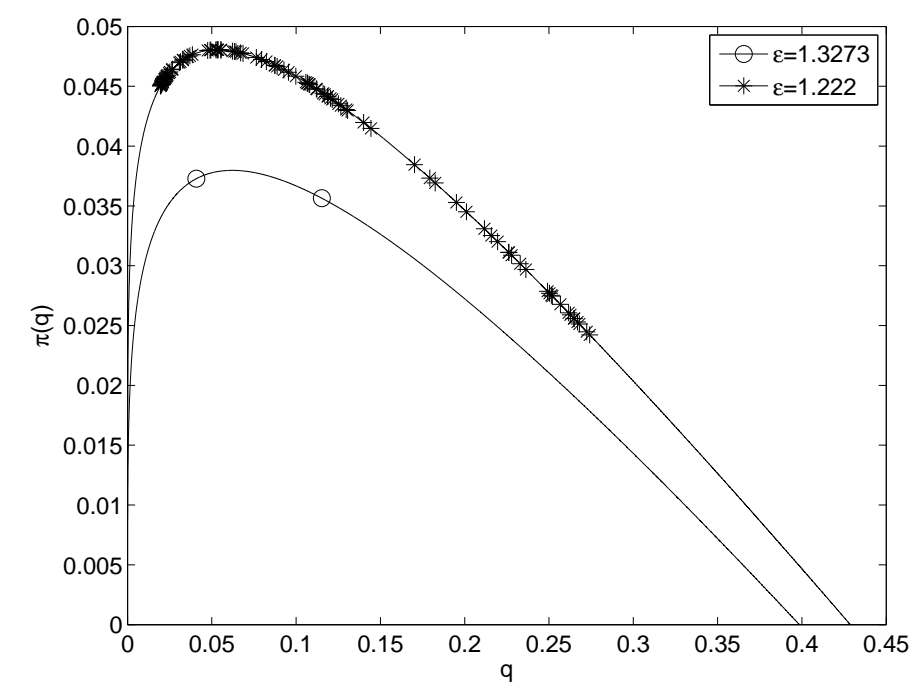

Figure 8: Profit functions for two different elasticity values, for which equilibrium is unstable. For the largest value of $\varepsilon$, the attractor consists of two points, while for the smallest value of $\varepsilon$ we have a chaotic attractor. In both cases, corresponding profits, respectively represented by circles and stars, are always smaller than equilibrium profits.

[13] Matsumoto A, Chiarella C, Szidarovszky F. Dynamic monopoly with bounded continuously distributed delay. Chaos, Solitons Fractals $2013 ; 47: 66-72$.

[14] Al-Hdaibat B, Govaerts W, Neirynck N. On periodic and chaotic behavior in a two-dimensional monopoly model. Chaos, Solitons Fractals 2015;70:27 -37 .

[15] Fanti L, Gori L, Sodini M. Nonlinear dynamics in a cournot duopoly with isoelastic demand. Math Comput in Simul 2013;doi: 10.1016/j.matcom.2013.09.004. Article in Press, available on line.

[16] Matsumoto A. Preferable disequilibrium in a nonlinear cobweb economy. Ann Oper Res 1999;89:101-23.

[17] Matsumoto A. Let it be: Chaotic price instability can be beneficial. Chaos, Solitons Fractals 2003;18(4):745-58.

[18] Matsumoto A. Controlling the cournot-nash chaos. J Optim Theory and Appl 2006;128(2):379-92. 


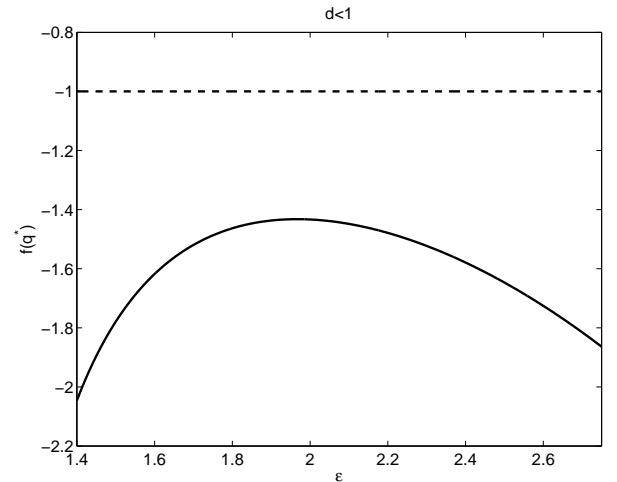

Figure 9: Plot of $f^{\prime}\left(q^{*}\right)$ on varying $\varepsilon$ for $a=0.1, c=0.23$. Equilibrium $q^{*}$ is never stable.
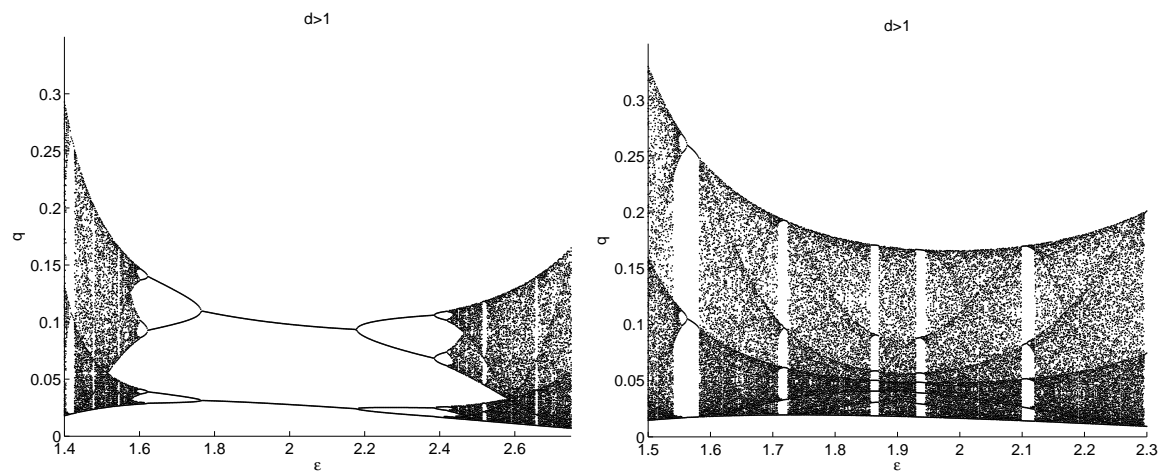

Figure 10: (Left plot) Bifurcation diagram for $a=0.1, c=0.23$. (Right plot) Bifurcation diagram for $a=0.1, c=0.24$. In both situations, trajectories of (5) never converge. In the former case, for some $\varepsilon$ we have periodic attractors, while in the latter the behavior is chaotic. 